\title{
Pore structure characterization based on joint numerical and experimental study: a case study of Nanpu Sag
}

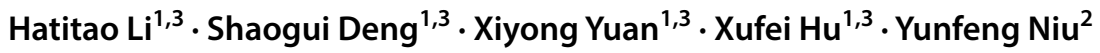

Received: 18 November 2018 / Accepted: 16 July 2019 / Published online: 22 July 2019

(c) The Author(s) 2019

\begin{abstract}
Petrophysical properties such as acoustic velocity, resistivity and nuclear magnetic resonance are greatly depending on the pore structures. This is because the pore structures could control the elastic modulus, conductive path and seepage distribution of the rock. The theoretical simulation and acoustic-electrical-nuclear magnetic resonance joint experiments were used to study the pore structure control action of acoustic, electrical and nuclear magnetic characteristics by taking the sandstone samples of Nanpu Sag as an example which the pore structure of the samples was classified based on the mercury intrusion fractal curve. It is found that the control action of pore structure type on the variation law of longitudinal and transverse wave velocity is mainly affected by the aspect ratio of pores; the effect of pore structure type on resistivity change is mainly affected by pore-throat ratio and tortuosity; the influence of pore structure on nuclear magnetic changes is mainly affected by pore size factors.
\end{abstract}

Keywords Pore structure $\cdot$ Control action · Petrophysical characteristics · Theoretical simulation - Acoustic-electricalnuclear magnetic resonance joint experiments

\section{Introduction}

Mercury intrusion, CT scan, nuclear magnetic resonance and other experiments have proved that the pore structure is expressed as a series of pores and throats of different sizes and shapes (Wu et al. 2017; Liu et al. 2009; Zhao et al. 2003; Liu et al. 2016; Ge et al. 2011). Rock pore structure affects the response characteristics of acoustic-electrical-nuclear magnetic logging (Guo et al. 2017, 2018). Prasad's (2001) research shows that the shape of the pore changes the speed of sound and the modulus of elasticity by affecting the propagation path of the wave. Kumar and Han (2005) believe that the increase of spherical pore content causes a large difference between the model sound velocity and the Wiley

Shaogui Deng

854938905@qq.com

1 School of Geosciences, China University of Petroleum, Qingdao 266580, China

2 Institute of Electronics, Chinese Academy of Sciences, Beijing 100190, China

3 Laboratory for Marine Mineral Resources, Qingdao National Laboratory for Marine Science and Technology, Qingdao 266071, China time average sound velocity. Verwer et al. (2011) combined with flakes and electrical experiments found that under the same porosity conditions, the greater the number of pores, the greater the cementation index, and it is believed that in carbonate rocks, small pores and complex pore structure reduce the cementation index. Xiao (2012) obtains the NMR response under certain magnetic conditions Based on the rock particle accumulation model, Monte Carlo and random walk algorithm, Liu et al. (2013) used the tube model to numerically simulate the conductive properties of different pore structures and proved that the pore structure is one of the factors leading to the divergence of the rock electricity experimental data. In this paper, the sandstone samples in Nanbao Sag are taken as an example. The basic parameters are given in Table 1. Then, the pore structure is classified based on the morphology of mercury intrusion curve. The classification results are shown in Fig. 1, and then, the control action of pore structure on the acoustic, electrical and nuclear magnetic characteristics of rock is studied based on theoretical simulation and acoustic-electromagnetic-nuclear magnetic combined experiments. 
Table 1 Sample basic parameters

Samples Well Depth (m) Porosity (\%) Permeability (mD)

1-13 X1 $3471.5-4154.7 \quad 6.5-14.9 / 11.2 \quad 0.289-2.881 / 1.411$

14-22 X2 3217.6-3356.5 $15.2-23.1 / 18.4 \quad 0.94-9.785 / 3.558$

$23-34 \quad \mathrm{X} 3 \quad 4214.1-5068.4 \quad 2.9-5.9 / 4.8 \quad 0.039-1.189 / 0.412$

\section{Acoustic velocity characteristics of sandstones with different pore structures}

The shape of the pores has a great influence on the velocity acoustic, and the increase of the proportion of spherical pores tends to increase the acoustic velocity. The study of acoustic velocity characteristics of sandstones with different pore structures has a great significance for the evaluation of porosity and gas-bearing identification using sonic logging data.
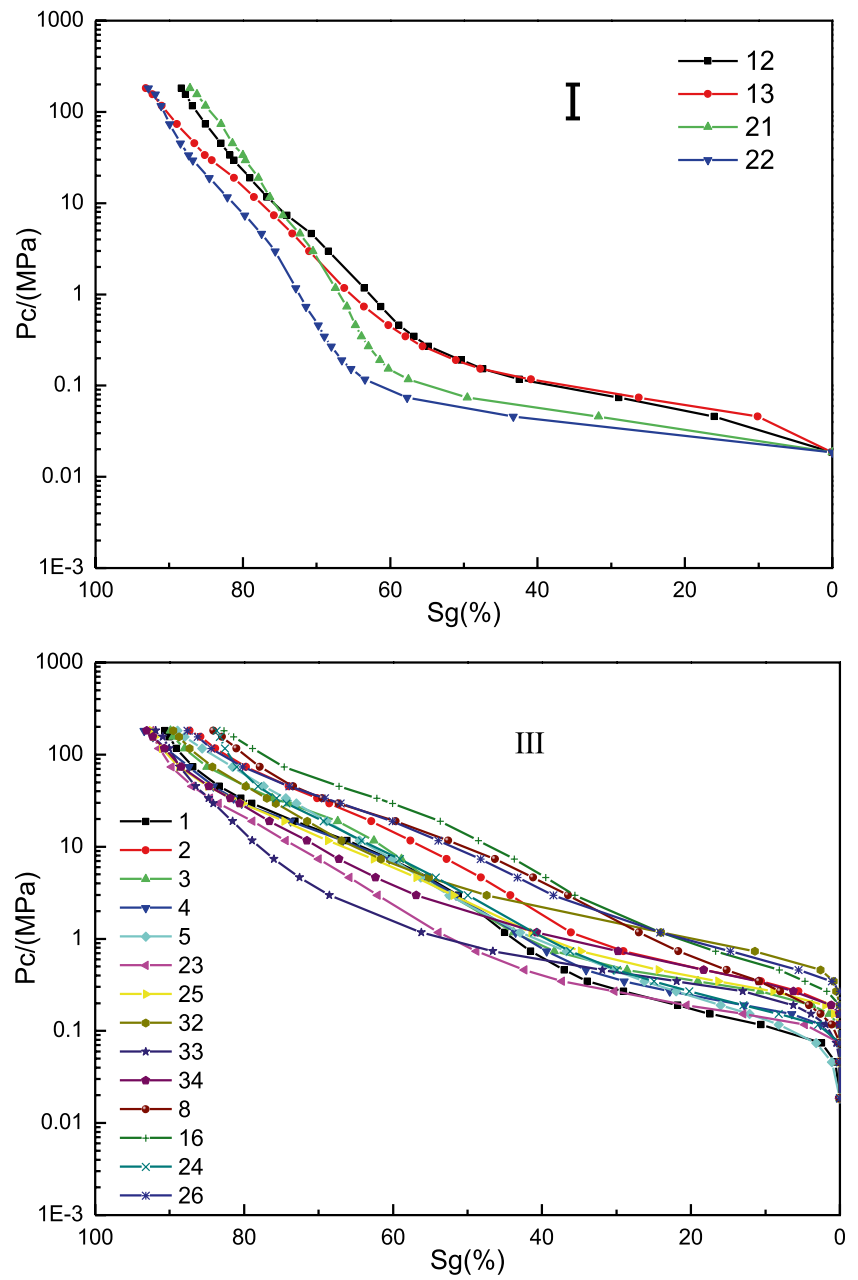

\section{Theoretical simulation of acoustic velocity based on DEM equation}

In order to study the effect of pore shape on the acoustic velocity of rock, the acoustic velocity of different pore aspect ratios is simulated based on differential equivalent medium (DEM) theory. The differential equivalent medium model is widely used in the study of elastic properties of porous media. Li and Zhang (2014) derived the analytical formula for the volume and shear modulus of multiple pore rocks under dry conditions.

$$
K_{\mathrm{dry}}=K_{\mathrm{m}} \frac{(1-\Phi) \sum_{i=1}^{N} v_{i} S_{0 i}\left[S_{1 i}+S_{2 i}-S_{3 i}+\frac{\left(S_{1 i}-S_{3 i}\right)\left(S_{2 i}-S_{3 i}\right)}{S_{3 i}+K_{\mathrm{m}} / G_{\mathrm{m}}}\right]}{\left[1+\frac{b K_{\mathrm{m}}}{a G_{\mathrm{m}}}-\frac{b K_{\mathrm{m}}}{a G_{\mathrm{m}}}(1-\Phi)^{a}\right]^{\frac{\sum_{i=1}^{N} v_{i} S_{0 i}}{b}}}
$$
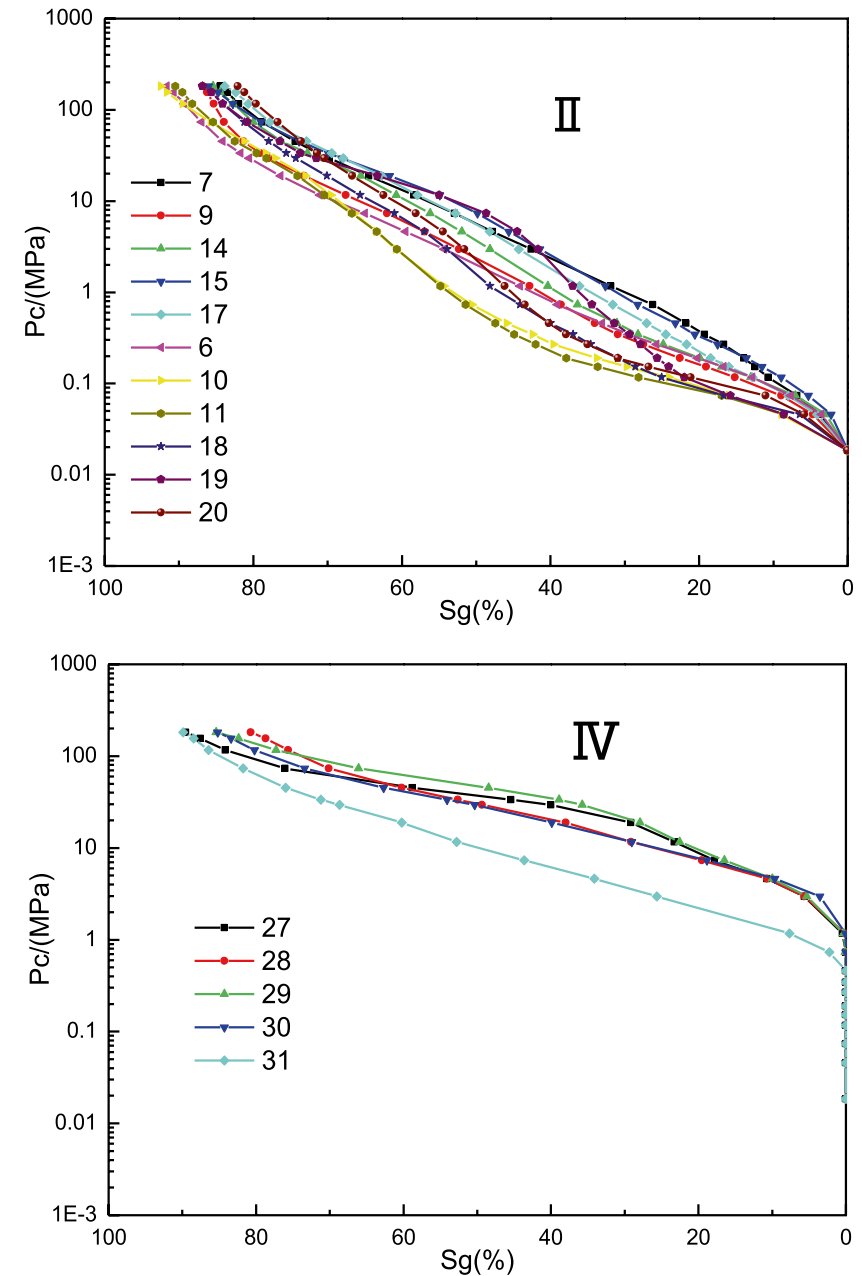

Fig. 1 Classification of pore structure based on mercury intrusion curve morphology 


$$
G_{\mathrm{dry}}=G_{\mathrm{m}} \frac{(1-\Phi) \sum_{i=1}^{N} v_{i} S_{0 i}\left[S_{1 i}+S_{2 i}-S_{3 i}+\frac{\left(S_{1 i}-S_{3 i}\right)\left(S_{2 i}-S_{3 i}\right)}{S_{3 i}+K_{\mathrm{m}} / G_{\mathrm{m}}}\right]^{-a}}{\left[1+\frac{b K_{\mathrm{m}}}{a G_{\mathrm{m}}}-\frac{b K_{\mathrm{m}}}{a G_{\mathrm{m}}}(1-\Phi)^{a}\right]^{\frac{\sum_{i=1}^{N} v_{i} S_{0 i}}{b}-1}}
$$

where $a$ and $b$ are parameters related to the shape of the pore, and $K_{\mathrm{m}}$ and $G_{\mathrm{m}}$ are the volume and elastic modulus of the rock skeleton.

$$
\begin{aligned}
& S_{0 i}=\frac{2-3 g_{i}-3 \theta_{i}}{4 \theta_{i}-6 \theta_{i}^{2}-4 g_{i}}, \quad S_{1 i}=\frac{\theta_{i}-g_{i}}{2-3 g_{i}-3 \theta_{i}}, \\
& S_{2 i}=\frac{4}{3}, \quad S 3 i=\frac{2\left(\theta_{i}-g_{i}\right)}{3\left(2 \theta_{i}-3 \theta_{i}^{2}-2 g_{i}\right)} . \\
& \theta_{i}=\left\{\begin{array}{l}
\frac{\alpha_{i}}{\left(1-\alpha_{i}^{2}\right)^{3 / 2}}\left[\arccos \left(\alpha_{i}\right)-\alpha_{i} \sqrt{1-\alpha_{i}^{2}}\right], \quad\left(\alpha_{i}<1\right) \\
\frac{\alpha_{i}}{\left(\alpha_{i 2}-1\right)^{3 / 2}}\left[\alpha_{i} \sqrt{\alpha_{i}^{2}-1}-\operatorname{arcosh}\left(\alpha_{i}\right)\right], \quad\left(\alpha_{i}>1\right)
\end{array}\right. \\
& g_{i}=\frac{\alpha_{i}^{2}}{1-\alpha_{i}^{2}}\left(3 \theta_{i}-2\right),
\end{aligned}
$$

$\alpha_{i}$ is the aspect ratio of the aperture of the $i$ ellipsoidal pore.

Volume and shear modulus under complete water conditions can be obtained from the Gassmann equation (Biot 1956):

$K_{\text {sat }}=K_{\text {dry }}+\frac{\left(1-\frac{K_{\text {dry }}}{K_{\mathrm{m}}}\right)^{2}}{\frac{\Phi}{K_{\mathrm{f}}}+\frac{1-\Phi}{K_{\mathrm{m}}}-\frac{K_{\mathrm{dry}}}{K_{\mathrm{m}}^{2}}}$

$G_{\mathrm{sat}}=G_{\mathrm{dry}}$
Through the relationship between the acoustic velocity and the volume and elastic modulus, the compressional velocity and shear velocity of the rock under complete water conditions can be calculated:

$\left\{\begin{array}{l}K_{\mathrm{sat}}=\rho v_{\mathrm{p}}^{2}-\frac{4}{3} \rho v_{\mathrm{s}}^{2} \\ G_{\mathrm{sat}}=\rho v_{\mathrm{s}}^{2}\end{array}\right.$

For low-porosity and -permeability sandstone dominated by quartz, set $K_{\mathrm{m}}=44 \mathrm{GPa}, G_{\mathrm{m}}=44 \mathrm{GPa}$ and $K_{\mathrm{f}}=2.19 \mathrm{GPa}$. The simulation results of the relationship between the compressional velocity and shear velocity and the porosity under different aperture aspect ratios are shown in Fig. 2. Under the same porosity condition, the compressional velocity and shear velocity increase with the increase of the aspect ratio of the pore; under the condition of smaller pore aspect ratio ( $\alpha \leq 0.3$ ), the sensitivity of the compressional velocity and shear velocity to porosity decreases with increasing porosity; under larger pore aspect ratio conditions $(\alpha>0.3)$, the sensitivity law of the compressional velocity and shear velocity to porosity is reversed. Consider the actual situation of the formation $(\Phi<0.4)$, with the increase of the aspect ratio of the pores, the sensitivity of the longitudinal and transverse wave velocity to the porosity gradually decreases. The simulation results are in agreement with Kumar's simulation results (Kumar and Han 2005).

\section{Experimental study on acoustic velocity characteristics of sandstones with different pore structures}

In order to analyze the acoustic velocity characteristics of different pore structure sandstones, the 34 sandstones of the compressional velocity and shear velocity measurement
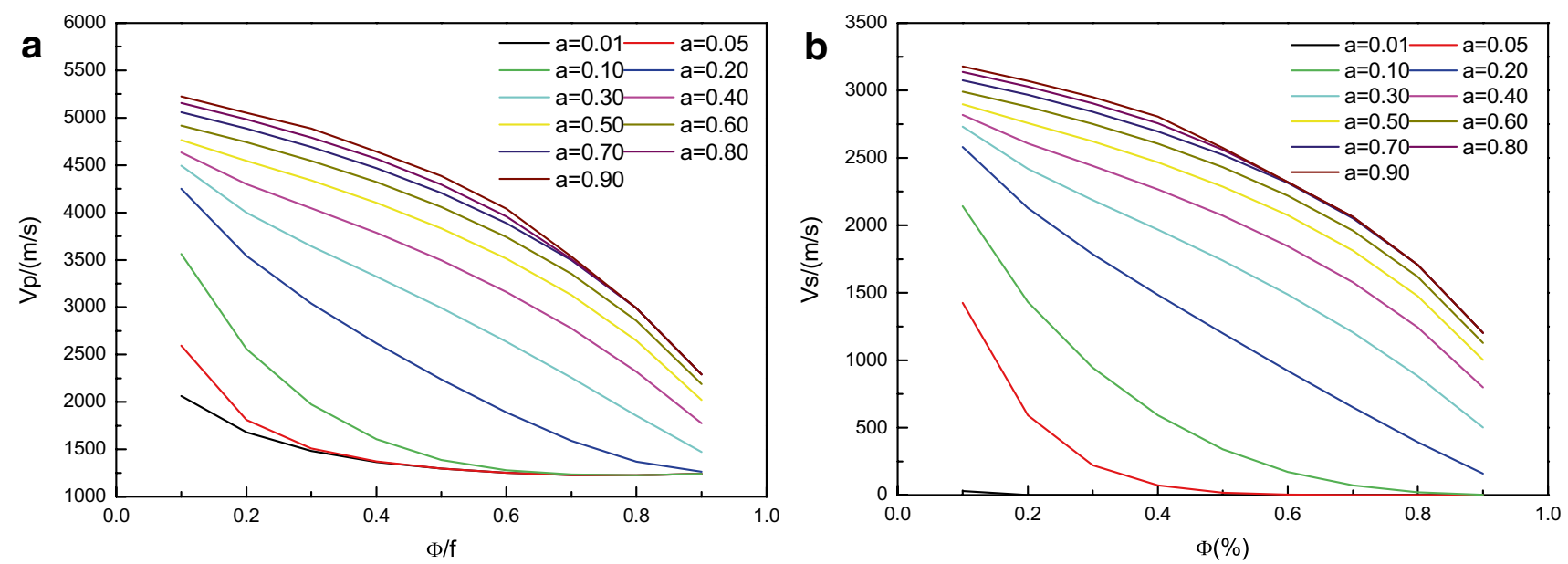

Fig. 2 a Theoretical relationship between $V_{\mathrm{p}}$ and $\Phi$; $\mathbf{b}$ theoretical relationship between $V_{\mathrm{s}}$ and $\Phi$ 
experiments under different water saturation conditions were carried out, and the results of the slice image processing of the corresponding rocks were obtained to obtain the longitudinal and transverse waves under different pore aspect ratio conditions. The relationship between acoustic velocity and porosity is shown in Fig. 3. It can be seen from the figure that the compressional velocity and shear velocity are inversely proportional to the porosity. The relationship between the two is linear in the experimental data. Under the same porosity condition, the compressional velocity and shear velocity increase with the increase of the aspect ratio of the pore. The variation of the data is consistent with the simulation results of Fig. 2.

According to the classification results of rock pore structure, the relationship between the compressional velocity and shear velocity and porosity of sandstones with different pore structures is obtained, as shown in Fig. 4. As the porosity increases, the compressional velocity and shear velocity decrease, and the type of pore structure controls the degree of decreasing trend. The pore structure is from type I to type IV, the proportion of macropores is gradually reduced, the trend of the compressional velocity and shear velocity decreases with the increase of porosity, and the sensitivity of the compressional velocity and shear velocity to the change of porosity is enhanced. This may be related to the variation of the aspect ratio of the pores of different pore structures. The average pore aspect ratios of the type I to type IV pore structure rocks are 0.251 , $0.248,0.237$ and 0.226 , respectively, which is consistent with the simulations of Figs. 2 and 3.
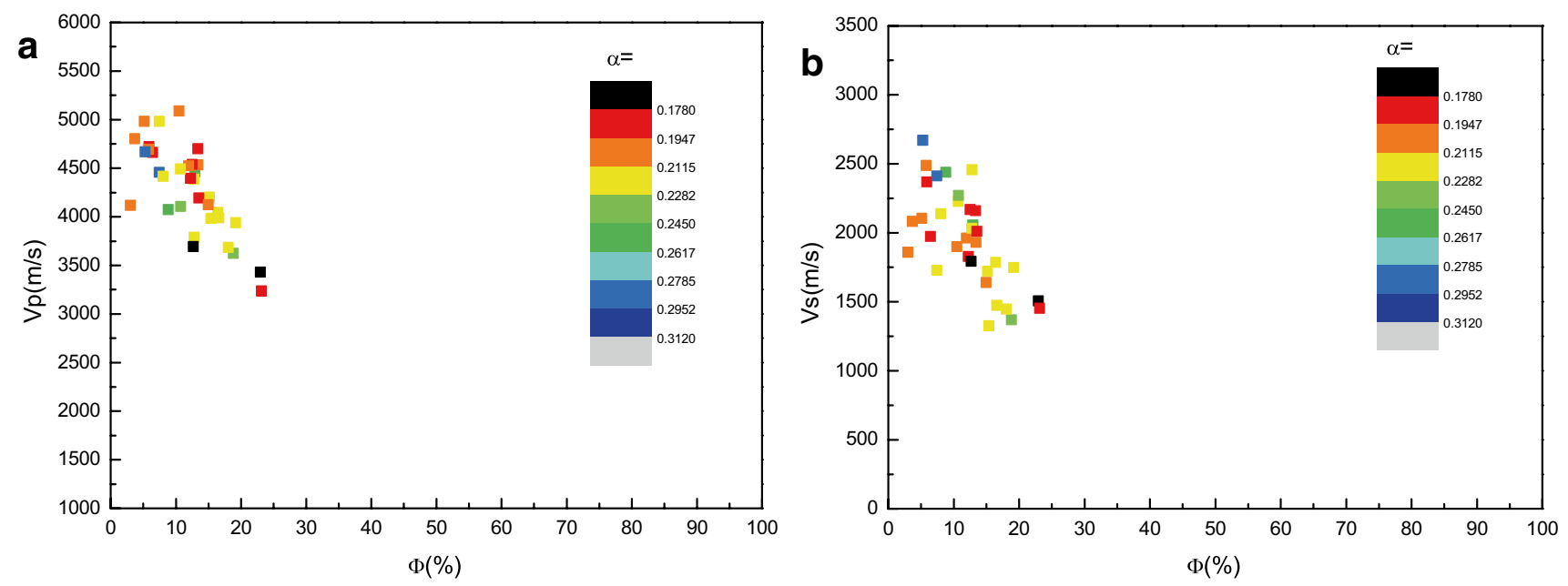

Fig. 3 a Experimental relationship between $V_{\mathrm{p}}$ and $\Phi ; \mathbf{b}$ experimental relationship between $V_{\mathrm{s}}$ and $\Phi$
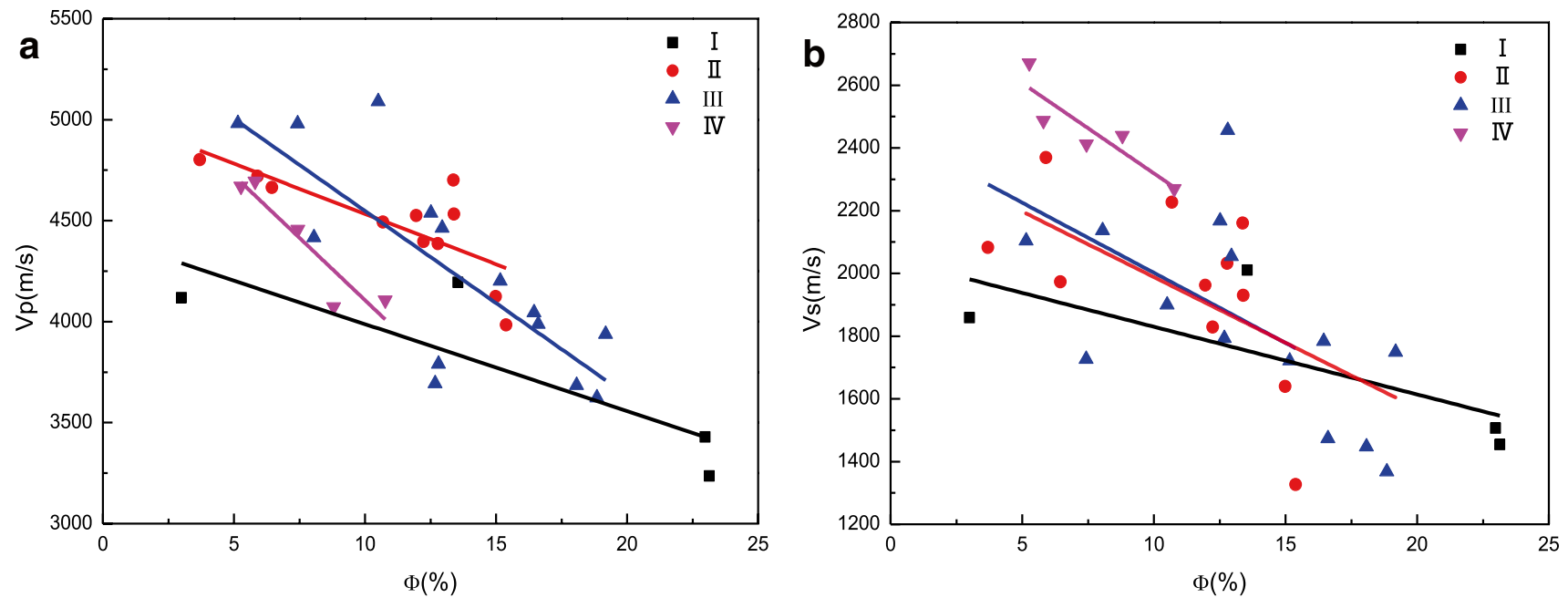

Fig. 4 a Crossplot of $V_{\mathrm{p}}$ and $\Phi$ in different pore structure sandstones; $\mathbf{b}$ crossplot of $V_{\mathrm{s}}$ and $\Phi$ in different pore structure sandstones 


\section{Resistivity characteristics of sandstones with different pore structures}

At present, resistivity is still the main means of calculating formation water saturation. Rock resistivity characteristics are closely related to pore structure. Rocks with the same porosity and different pore structures tend to exhibit different resistivity characteristics. Several key parameters of Archie's formulas are also affected by the pore structure. Therefore, studying the resistivity characteristics of sandstones with different pore structures has great significance for fluid identification and calculation of formation water saturation.

\section{Theoretical simulation of influence of pore structure on resistivity characteristics}

(1) Influence of pore-throat ratio and tortuosity on formation factor

To investigate the effect of pore-throat ratio and tortuosity on formation factor, a simplified bulb model can be constructed to characterize the pores, as shown in Fig. 5. The pore body comprises two parts, a pore and a throat. The pore can be regarded as composed of a plurality of pore-throat units, and the resistivity of one pore-throat unit can be expressed as:

$r_{\mathrm{u}}=R_{\mathrm{w}}\left[\frac{3}{\pi r}+\frac{\tau L \mathrm{Par}^{2}}{\pi r^{2}}\right]$

where $R_{\mathrm{w}}$ is the formation water resistivity; $A_{\mathrm{m}}$ and $L_{\mathrm{m}}$ are the cross-sectional area and length of the pore conduction direction, respectively; $\tau$ is the tortuosity of the throat; $r$ is the radius of the sphere; $L$ is the length of the throat in the conductive direction; and Par is the pore-throat ratio.

In the above pore network, it can be considered that the pore resistivity is the result that the pore-throat unit is $T$ connected in parallel and then in series connection, and the

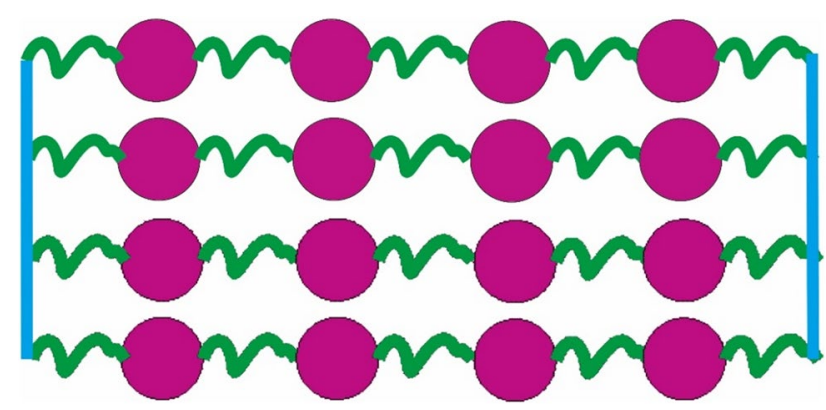

Fig. 5 A simplified tube model resistivity of the saturated water system is obtained according to Ohm's law:

$R_{0}=\frac{J}{N} R_{\mathrm{w}}\left[\frac{3}{\pi r}+\frac{\tau L P \mathrm{Par}^{2}}{\pi r^{2}}\right] \frac{A_{\mathrm{m}}}{L_{\mathrm{m}}}$

where $N$ is the number of rows of the model; and $J$ is the number of pore-throat units in the model.

The porosity of the model can be expressed as:

$\Phi=\frac{\left(\frac{4}{3} \pi r^{3}+\pi r^{2} \tau L \operatorname{Par}^{2}\right) N J}{A_{\mathrm{m}} L_{\mathrm{m}}}$

Setting $2 r=L$, according to the Archie formula, the following can be obtained:

$F=\frac{J^{2}}{16 N^{2} \Phi}\left(3+2 \tau \operatorname{Par}^{2}\right)\left(\frac{4}{3}+2 \tau \operatorname{Par}^{2}\right)$

where $F$ is the formation factor.

The relationship between Par, $\tau, F$ and is $\Phi$ shown in Fig. 6. It can be seen from the figure that formation factors increase with the increase of the pore-throat ratio. Under relatively low-porosity conditions, the influence of pore-throat ratio on the formation factors is greater, and the reason may be: under low-porosity conditions, the larger the pore-throat ratio, the more complex the pore structure, the more rugged the conductive path, and the greater the pore-throat ratio, the partial throat may be closed, thus resulting in a faster increase of pore resistivity. Formation factor is positively correlated with the degree of tortuosity, and the larger the pore-throat ratio, the greater the influence on formation factors. Figure 7 shows the simulation results of formation factor under different porosity and pore-throat ratios, different hole-throat ratios and tortuosity. As shown in the figure, under the condition of fixed tortuosity, the larger the pore-throat ratio and the smaller the porosity is, the larger the formation factor is; under the condition of fixed porosity, the greater the tortuosity and the larger the pore-throat ratio is, the larger the formation factor is.

(2) $I-S_{\mathrm{w}}$ relationship in a two-pore-component model

In order to study the relationship between the resistivity increase rate I and the water saturation $S_{\mathrm{w}}$ of different pore structure rocks, the data were fitted by the double-pore-component model and the Archie model, and the effect of pore structure on electrical conductivity is studied by comparing difference between the two fitting curves (Li et al. 2018; Shi et al. 2008). The two-pore-component model is shown in Fig. 8, the pores are divided into macropores and micropores. The macropores are equivalent to movable pores, and the small pores are equivalent to dead pores. The model

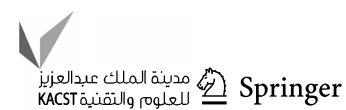



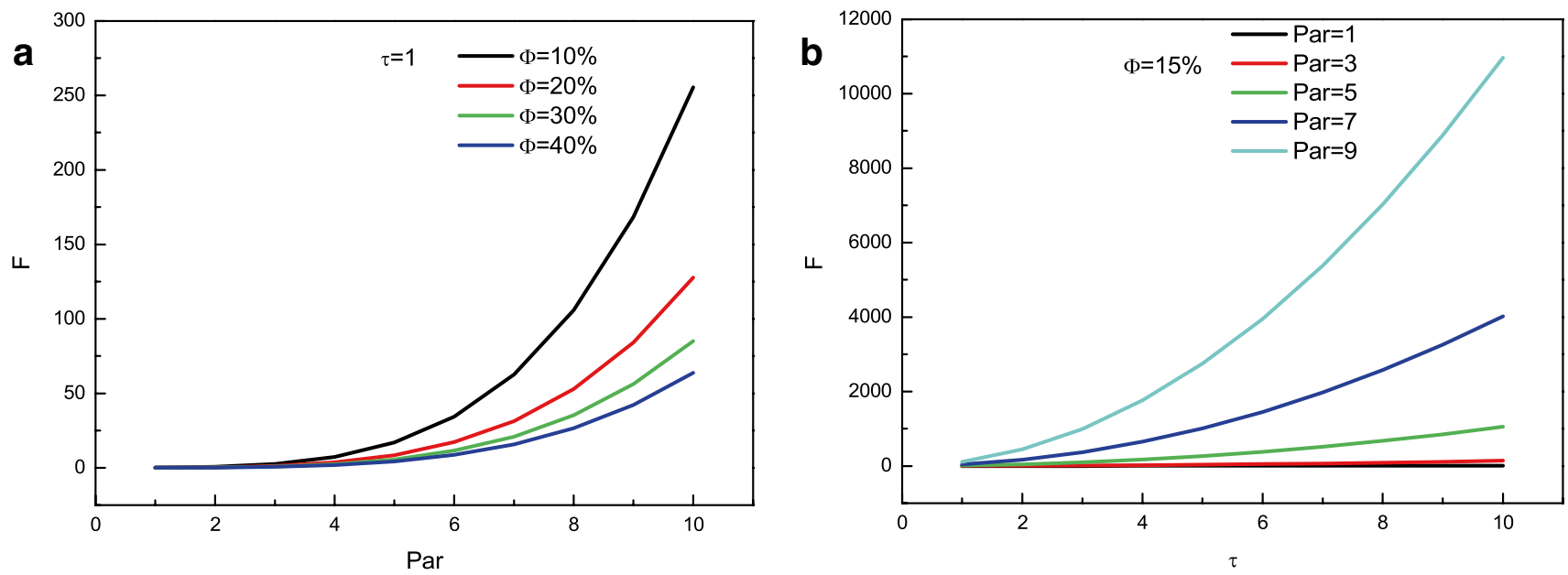

Fig. 6 a $F$ and pore-throat ratio; $\mathbf{b} F$ and tortuosity
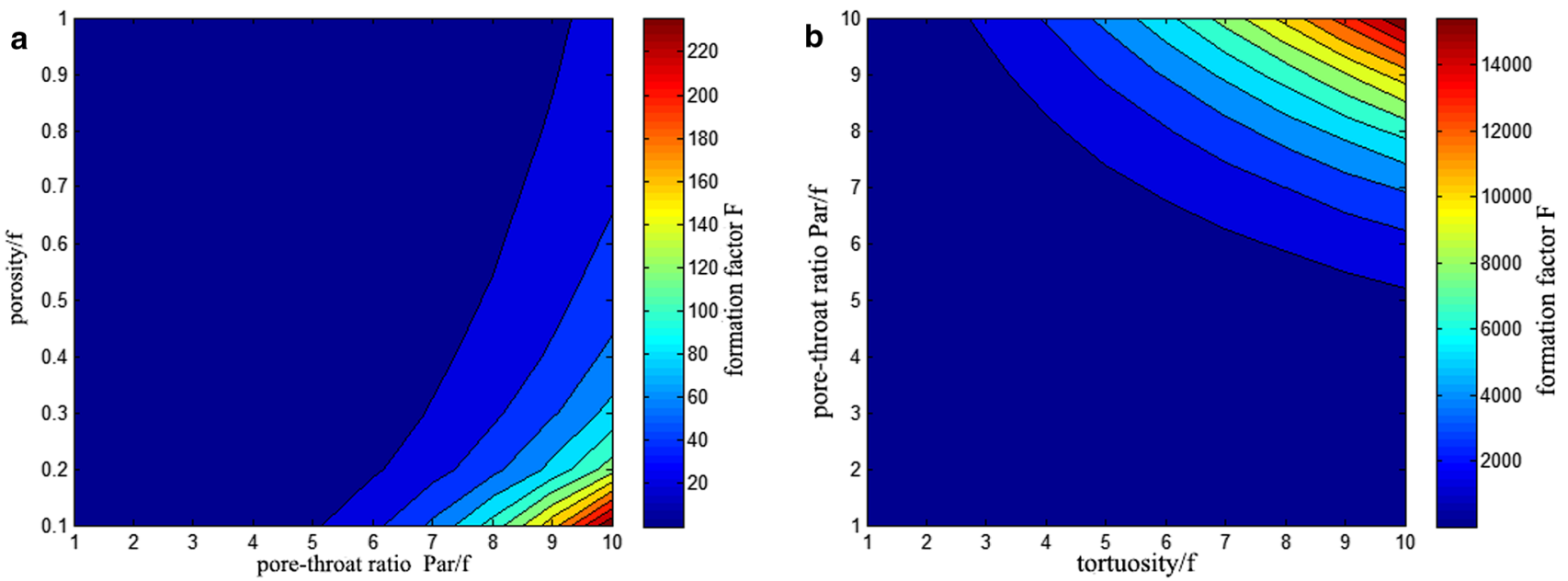

Fig. 7 a $F$ under different porosity and pore-throat ratios; $\mathbf{b} F$ under different hole-throat ratios and tortuosity

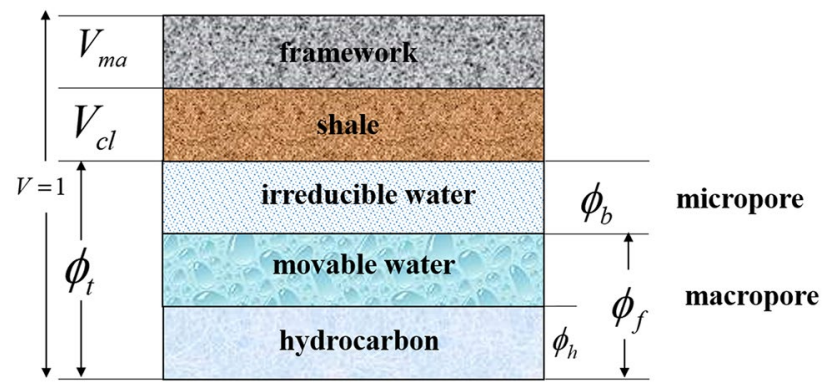

Fig. 8 Physical model of the dual-pore component rock physical volume

considers that the resistivity of a rock can be equivalent to the result of a three-part resistance in parallel: (1) conductive fluid in large pores; (2) bound water in the remaining connected pores; and (3) Skeletal conduction caused by muddy and conductive minerals. According to previous research results, in this model, the relationship between $I$ and $S_{\mathrm{w}}$ can be expressed as (Li et al. 2012):

$$
\begin{aligned}
& I=\frac{1}{\frac{\phi_{\mathrm{f}}^{\mathrm{mf}}}{\phi_{\mathrm{f}}^{\mathrm{mf}}+\phi_{\mathrm{b}}^{\mathrm{mb}}} S_{\mathrm{wf}}^{\mathrm{n}}+\frac{\phi_{b}^{\mathrm{mb}}}{\phi_{\mathrm{f}}^{\mathrm{mf}}+\phi_{\mathrm{b}}^{\mathrm{mb}}}} \\
& S_{\mathrm{w}}=\frac{\phi_{\mathrm{f}} S_{\mathrm{wf}}+\phi_{\mathrm{b}}}{\phi_{\mathrm{t}}}
\end{aligned}
$$

where $\phi_{\mathrm{f}}$ and $\phi_{\mathrm{b}}$ are the porosity of the two pore components; $m_{\mathrm{f}}$ and $m_{\mathrm{b}}$ are the cementation index of the two pore components, respectively.

In the rock of different pore structure types, one sample was selected as the representative, and the resistivity data of the four rocks were fitted using the Archie and the 

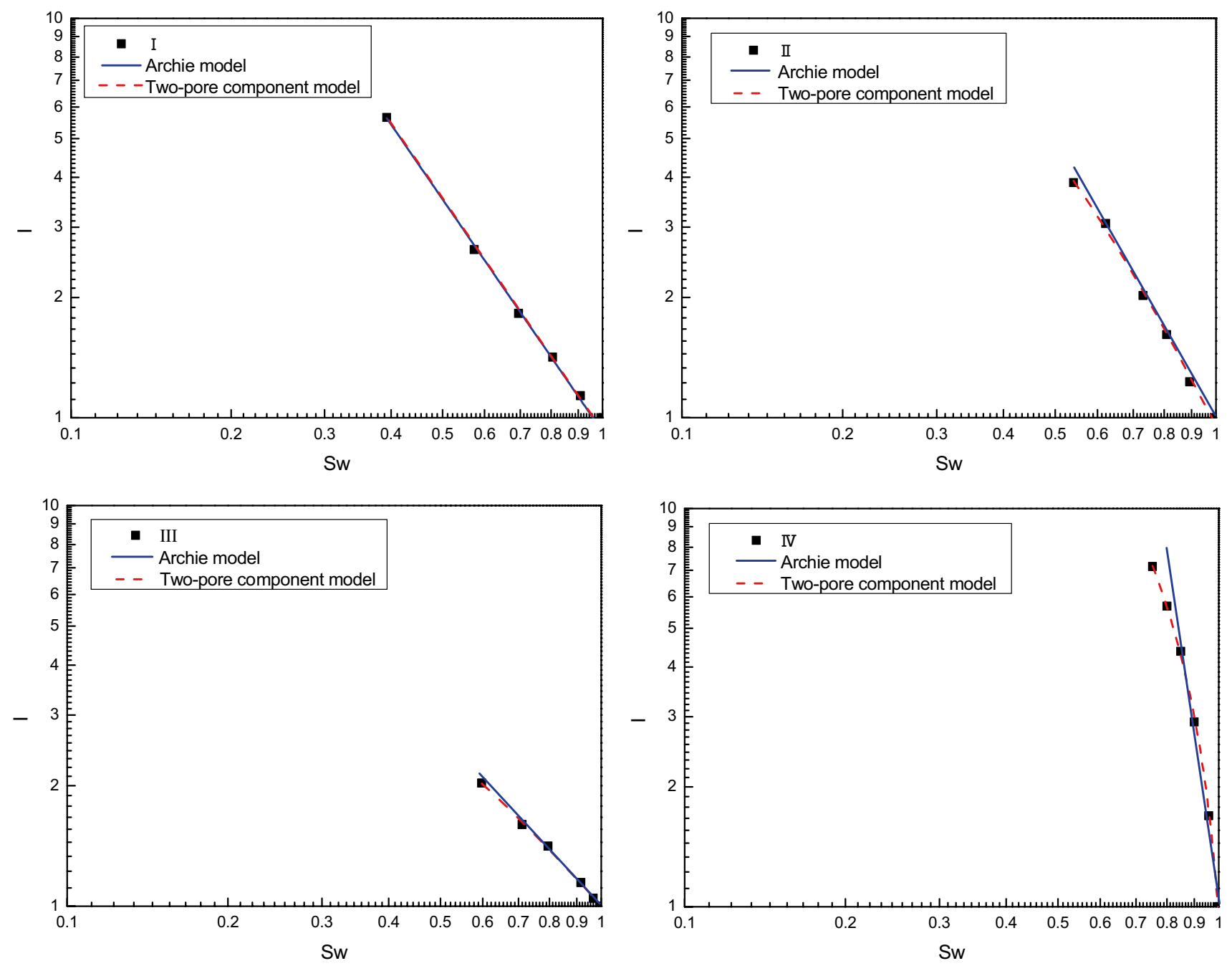

Fig. 9 Fitting results of resistivity data for two models

two-pore-component models, respectively, as shown in Fig. 9. For the type I pore structure samples with large pores, the fitting results of the two models overlap, indicating that the water saturations evaluated by the two models are more accurate in the macroporous-based formation. The proportion of large pores of type II pore structure rocks decreases slightly. When $S_{\mathrm{w}}$ is close to the irreducible water saturation, the double-pore-component model curve is slightly lower than the Archie model fitting curve. In this case, the results of the two models are less different, and the proportion of large pores of type III pore structure rocks decreases further. Near the irreducible water saturation, the double-porecomponent model is much lower than the Archie model. In this case, the double-pore-component model is preferred for water saturation evaluation; type IV pore structure rocks are mainly small pores, and the irreducible water saturation is about $70 \%$. In the vicinity of the irreducible water saturation, the double-pore-component model is much lower than the Archie model. In this case, $\mathrm{Al}$ is used. The calculation of water saturation in the odd model will have a large deviation from the actual situation, which leads to the wrong interpretation.

\section{Experimental analysis of resistivity characteristics of rocks with different pore structures}

In order to study the resistivity characteristics of sandstones with different pore structures, rock resistivity measurement experiments were carried out for 34 sandstones in the study area under different water saturation conditions. The salinity of the solution used in the experiment was $4000 \mathrm{ppm}$. The relationship between formation factors and porosity of four different pore structure rocks is shown in Fig. 10. The rock pore structure is from type I to type IV, and the slope of $F-\Phi$ in the double logarithmic coordinate system is gradually reduced, that is, the cementation index is gradually 


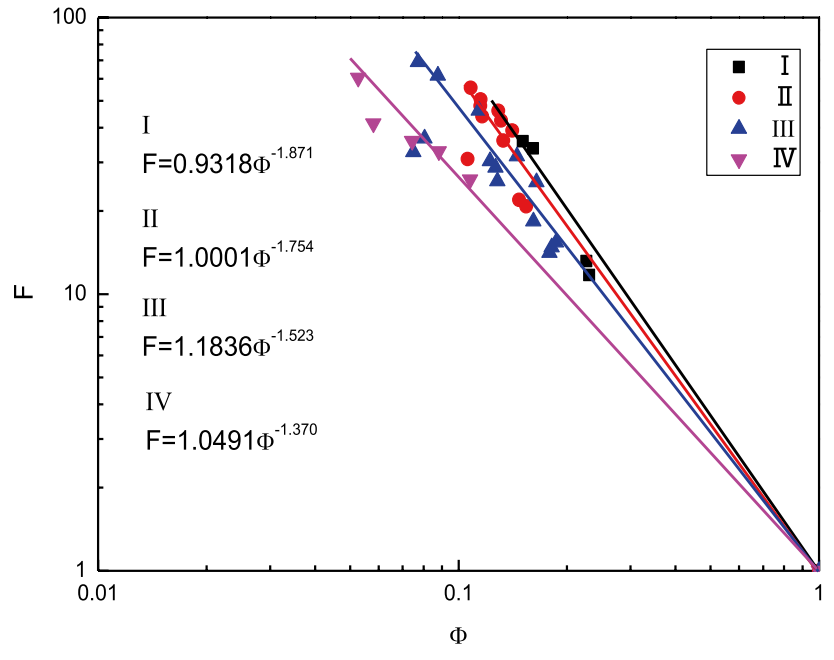

Fig. $10 F-\Phi$ relationship diagram of rocks with different pore structures

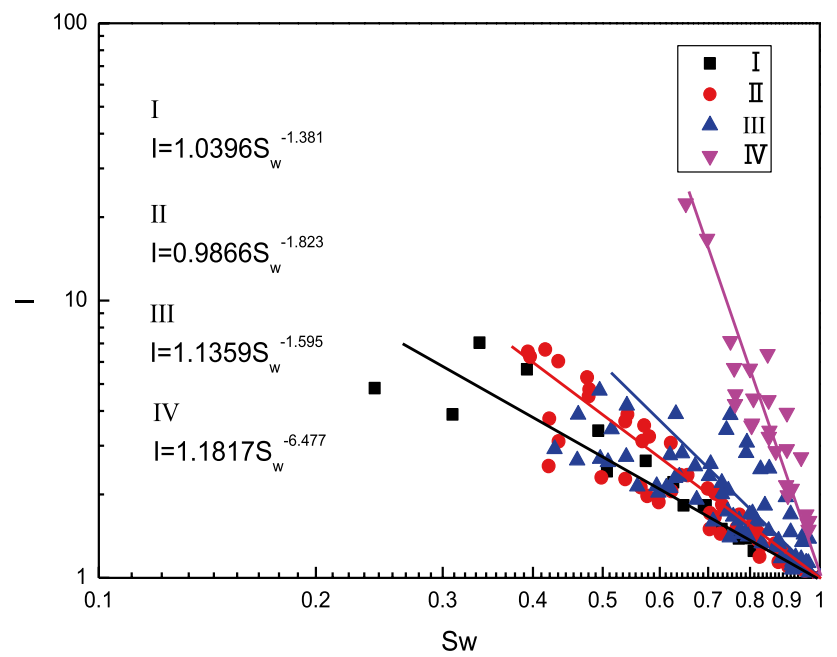

Fig. $11 I-S_{\mathrm{w}}$ relationship of different pore structure rocks

decreased, and under the same porosity condition, the value of $F$ is gradually decreased, and the pore throat is gradually reduced. It gradually decreases from the average value, and this phenomenon is consistent with the previous simulation conclusion.

The relationship between the resistivity increase rate and the water saturation of four different pore structure rocks is shown in Fig. 11. The slope of the straight line of $I-S_{\mathrm{w}}$ in the double logarithmic coordinate system is gradually increased in the rock pore structure from type I to type IV.

The relationship between the cementation index of different pore structure rocks and the porosity and pore radius is shown in Fig. 12. The cementation index $m$ increases first and then decreases with the increase of porosity and pore radius. The variation of porosity and pore radius rocks is different in four types of pore structure, and the variation of $m$ is different. The relationship between the saturation index of different pore structure rocks and the porosity and pore radius is shown in Fig. 13. The saturation index $n$ decreases with the increase of porosity and pore radius and the two approximate power functions.

\section{Nuclear magnetic resonance characteristics of rocks with different pore structures}

Nuclear magnetic resonance logging is an important tool to evaluate the pore structure characteristics of rock. The characteristics of pore distribution determine the morphology of nuclear magnetic resonance $T_{2}$ spectrum. The geometric mean value of nuclear magnetic resonance $T_{2}$, cutoff value and the key parameters of nuclear magnetic resonance such as irreducible water saturation also have an effect. Therefore, it is a great significance to evaluate the influence of pore structure on nuclear magnetic resonance characteristics through simulation and experimental research.

\section{Construction of $T_{2}$ spectrum of rocks with different pore structures}

In order to study the influence of pore structure type on the morphology and nuclear magnetic resonance parameters of $T_{2}$ spectrum, the nuclear magnetic resonance $T_{2}$ spectrum of different pore structure rocks was constructed based on the pore size distribution of different pore structure rocks and the mercury intrusion curve characteristics of various pore structure rocks. The porosity is set to $12 \%$, the echo interval is $0.2 \mathrm{~ms}$, and the number of echoes is 2048 . The nuclear magnetic attenuation spectra of rocks with different pore structures are obtained, as shown in Fig. 14.

The modified SVD inversion method was used to invert the above-mentioned nuclear magnetic attenuation spectrum, and the nuclear magnetic resonance $T_{2}$ spectrum of different pore structure rocks was obtained, as shown in Fig. 15 (Gao and Xiao 2015). The $T_{2}$ spectrum of the type I pore structure rock is a typical bimodal, with large pores as the main type, and the typical bimodal coarse pore-throat type. The $T_{2}$ spectrum of the type II pore structure rock is bimodal too, mainly composed of small pores; it is a typical bimodal fine-thick pore-throat type; the $T_{2}$ spectrum of the type III pore structure rock has a single-peak shape, and the morphology is a single-peak coarse pore pore-throat type; the $T_{2}$ spectrum of the type IV pore structure rock has a single-peak shape, and the morphology is a unimodal pore-throat type The nuclear magnetic $T_{2}$ geometric mean, the $T_{2}$ arithmetic mean and the cutoff value calculated from the $T_{2}$ spectrum are given in Table 2. Under the same porosity conditions, the pore structure is from type I to type IV, and the $T_{2}$ geometric 

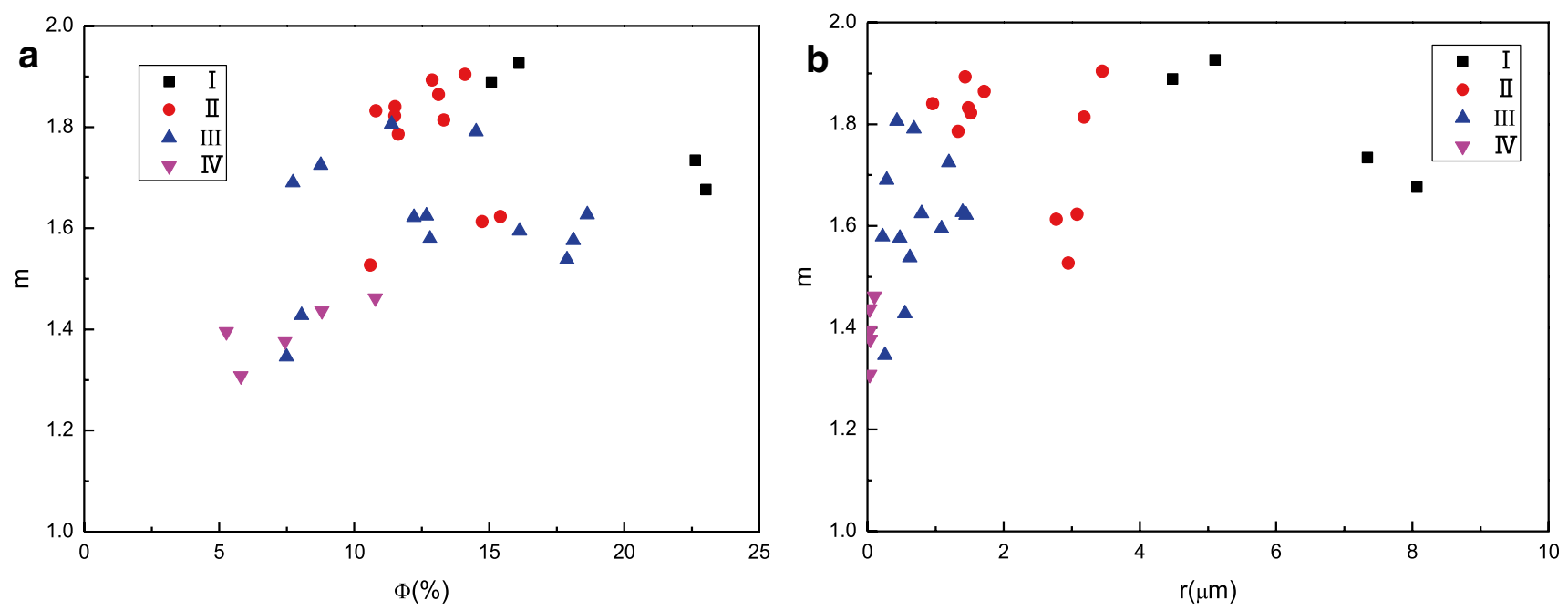

Fig. 12 a $m-\Phi$ relationship of rock with different pore structures; $\mathbf{b} m-r$ relationship of rock with different pore structures
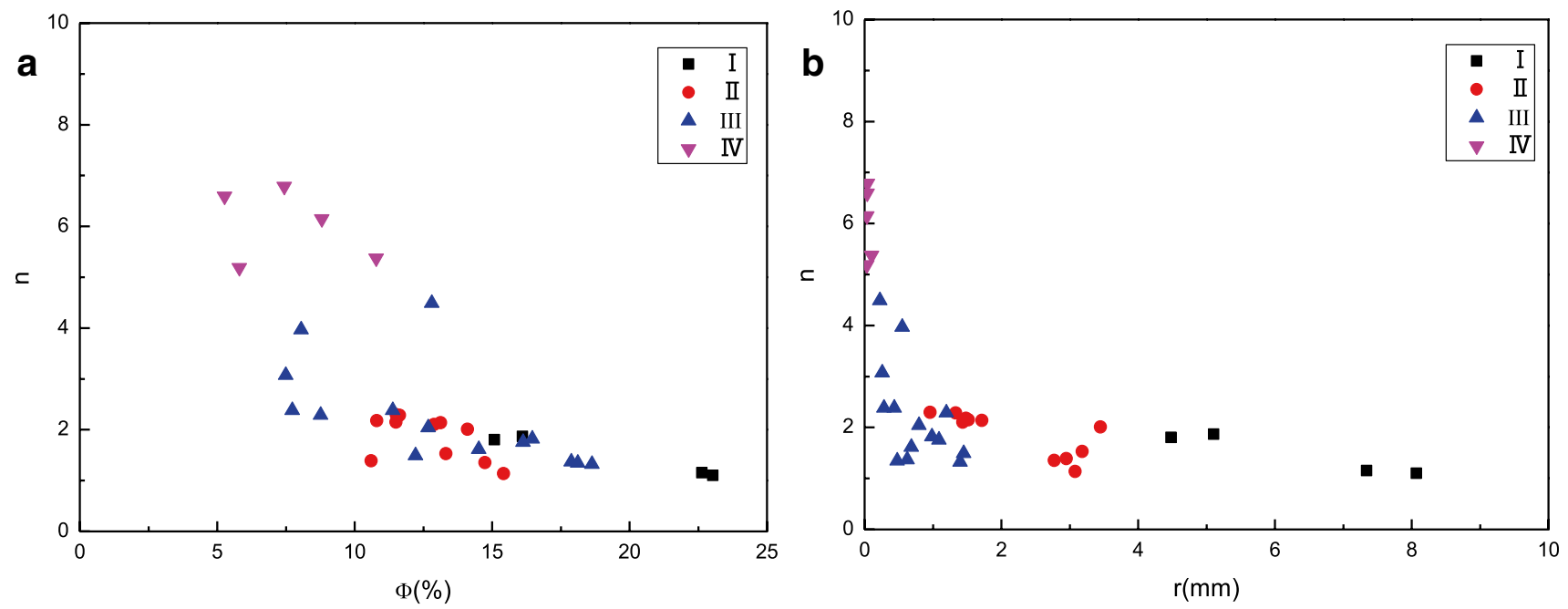

Fig. 13 a $n-\Phi$ relationship of rock with different pore structures; $\mathbf{b} n-r$ relationship of rock with different pore structures

mean and the arithmetic mean decrease, that is, the $T_{2}$ geometric and the arithmetic mean can be used to characterize the pore structure.

\section{Analysis of nuclear magnetic resonance experimental characteristics of rocks with different pore structures}

Aims to study the nuclear magnetic resonance characteristics of rocks with different pore structures, the $T_{2}$ spectrum experiment of nuclear magnetic resonance was carried out for 34 sandstones in the study area. The echo interval was set to $0.2 \mathrm{~ms}$, the waiting time was $6000 \mathrm{~ms}$ and the number of echoes was 2048. The formation water salinity at $4000 \mathrm{ppm}$, the nuclear magnetic $T_{2}$ spectrum of rocks with different pore structures under complete water conditions is obtained, as shown in Fig. 16. The $T_{2}$ spectrum of the type I pore structure rock is bimodal coarse-fine pore-throat type; the $T_{2}$ spectrum of the type II pore structure rock is bimodal fine-thick pore-throat type; the $T_{2}$ spectrum of the type III pore structure rock is mainly bimodal fine-rough hole-throat type and unimodal coarse hole-throat type; type IV pore structure rock $T_{2}$ spectrum is unimodal pore-throat type.

The fractal characteristics of mercury intrusion curves have been generally recognized. The fractal features of mercury intrusion curves have been widely used in pore structure classification and parameter calculation (Xiao 1998; Ma et al. 2004; Wei and Xiao 2009; Zhang et al. 2017). According to the relationship between the capillary pressure and the 

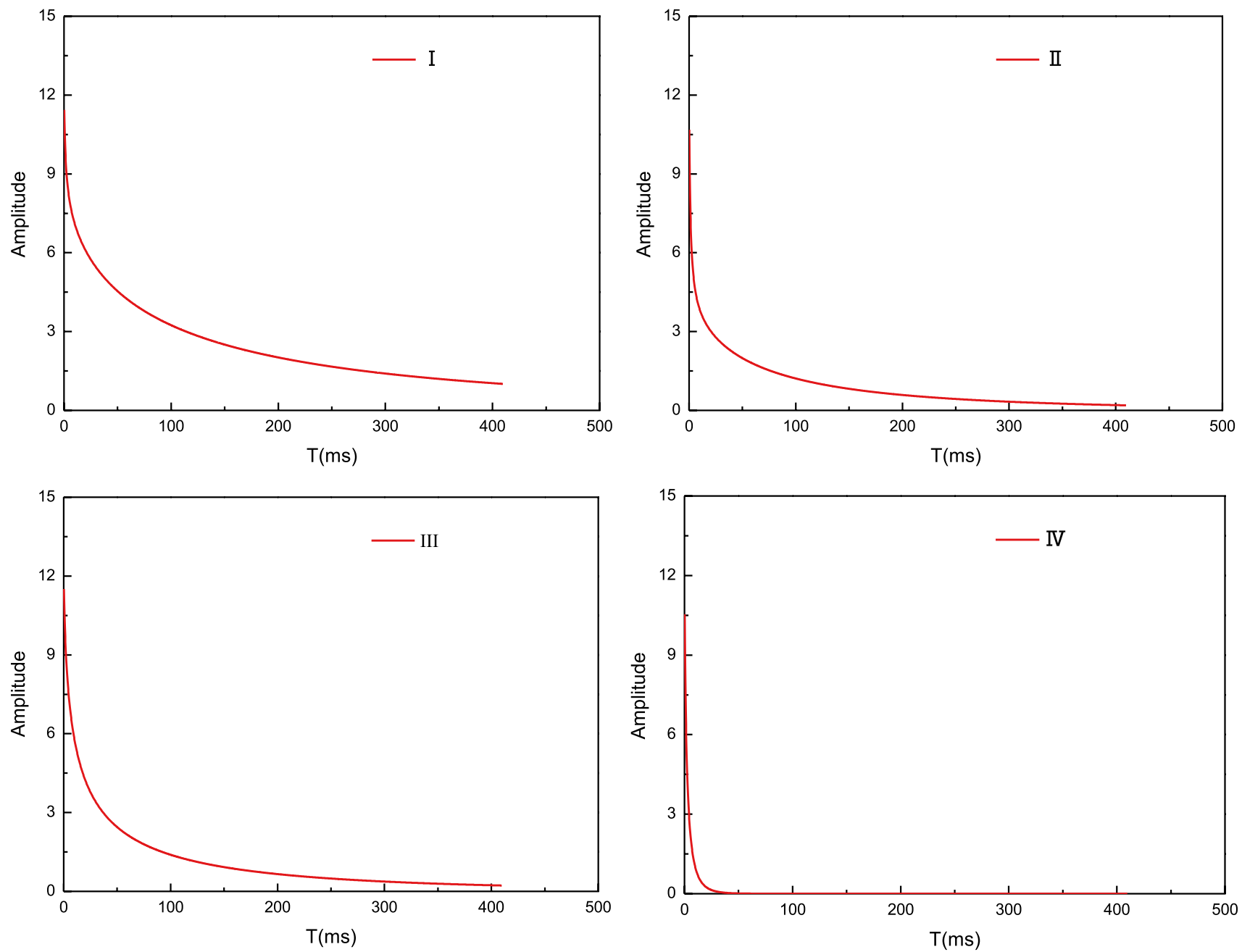

Fig. 14 Nuclear magnetic decay spectrum of rocks with different pore structures

$T_{2}$ value, the following formula can be obtained as (He et al. 2005; Xia et al. 2012):

$\lg \left(S_{\mathrm{v}}\right)=(3-D) \lg \left(T_{2}\right)+(D-3) \lg T_{2 \max }$

where $D$ is the fractal dimension, $S_{\mathrm{v}}$ is the cumulative water saturation, and $T_{2 \max }$ is the maximum amplitude in the NMR $T_{2}$ spectrum.

The above formula shows that the $T_{2}$ spectrum has fractal characteristics like the mercury intrusion curve. The cumulative water saturation $S_{\mathrm{v}}$ and $T_{2}$ values are linear in the double logarithmic coordinate system, and the fractal dimension can be obtained by using the slope. The nuclear magnetic $T_{2}$ spectrum fractal characteristic curves of samples 1 and 2 are shown in Fig. 17. In the figure, Dh 1 is the macroporous fractal dimension, and Dh2 is the small-pore fractal dimension. The fractal characteristic curve of $T_{2}$ spectrum has obvious multi-segment fractal features. The fractal dimension of small pores is smaller than that of large pores, and the fractal dimension ranges from 2 to 3 .
The relationship between the nuclear magnetic resonance $T_{2}$ geometric mean and macropore fractal dimension and porosity of different pore structure rocks is shown in Fig. 18. $T_{2 \mathrm{gm}}$ increases with the increase of porosity, and the $T_{2 \mathrm{gm}}$ sensitivity of pore structure to porosity from type I to type IV decreases gradually. The fractal dimension of macropores decreases with the increase of porosity from type I to type IV of pore structure. The sensitivity of $D h 1$ to porosity is gradually reduced.

\section{Conclusions}

Based on the theoretical simulation and combined acoustic and NMR experiments, the following can be obtained:

(1) Under the same porosity condition, the compressional velocity and shear velocity increase with the increase of the aspect ratio of the pore. Under the condition of 

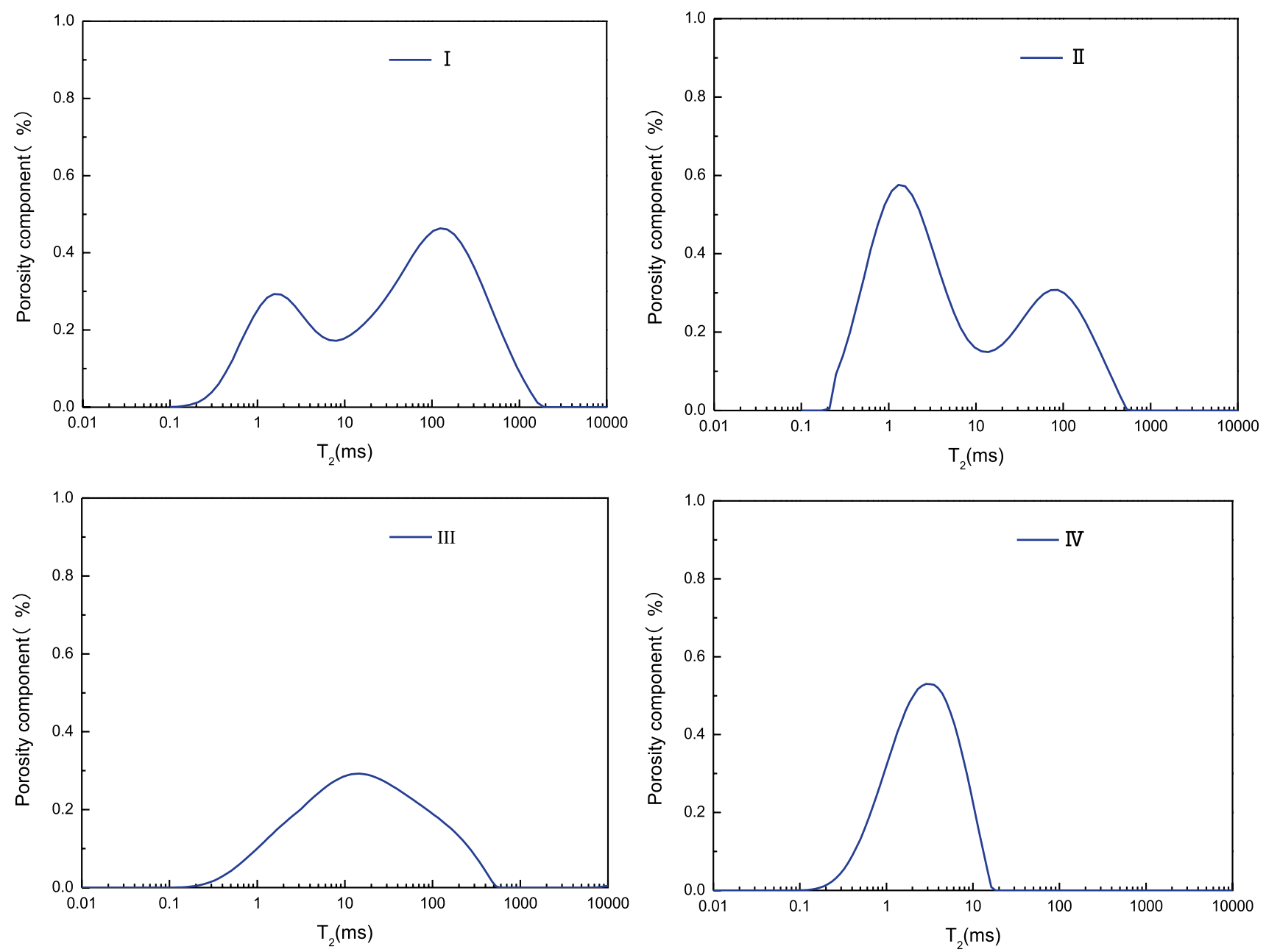

Fig. 15 Inversion of nuclear magnetic decay spectra with different pore structures

Table $2 T_{2}$ parameter table of rock structure spectrum with different pore structures

\begin{tabular}{lcc}
\hline Pore structure type & $T_{2}$ geometric mean $(\mathrm{ms})$ & $\begin{array}{l}T_{2} \text { arithmetic } \\
\text { mean }(\mathrm{ms})\end{array}$ \\
\hline I & 28.233 & 131.426 \\
II & 14.979 & 52.232 \\
III & 6.119 & 37.428 \\
IV & 2.459 & 3.484 \\
\hline
\end{tabular}

smaller pore aspect ratio, the sensitivity of the compressional velocity and shear velocity to the porosity is reduced; under the condition of larger pore aspect ratio, the variation of the sensitivity of the compressional velocity and shear velocity to the porosity is reversed; the control action of the pore structure type on the variation law of the compressional velocity and shear velocity is affected by the aspect ratio of the pore.
(2) The formation factors increase with the increase of the pore-throat ratio. The porosity-throat ratio has a greater influence on the smaller porosity, and the formation factor is positively correlated with the tortuosity. The larger the pore-throat ratio is, the greater the influence of the tortuosity on the formation factor is. In the pore structure dominated by small pores, the two-pore-component model should be used to fit the resistivity data to obtain the saturation model; there are differences in the variation laws of $m$ and $n$ in different pore structure rocks. The saturation index $n$ is approximately a power function with porosity and pore radius.

(3) The pore structure controls the morphology of the $T_{2}$ spectrum. From type I to type IV, the $T_{2}$ geometric mean and the arithmetic mean decrease. The sensitivity of $T_{2 \mathrm{gm}}$ and $D h 1$ to the change of porosity gradually decreases. 

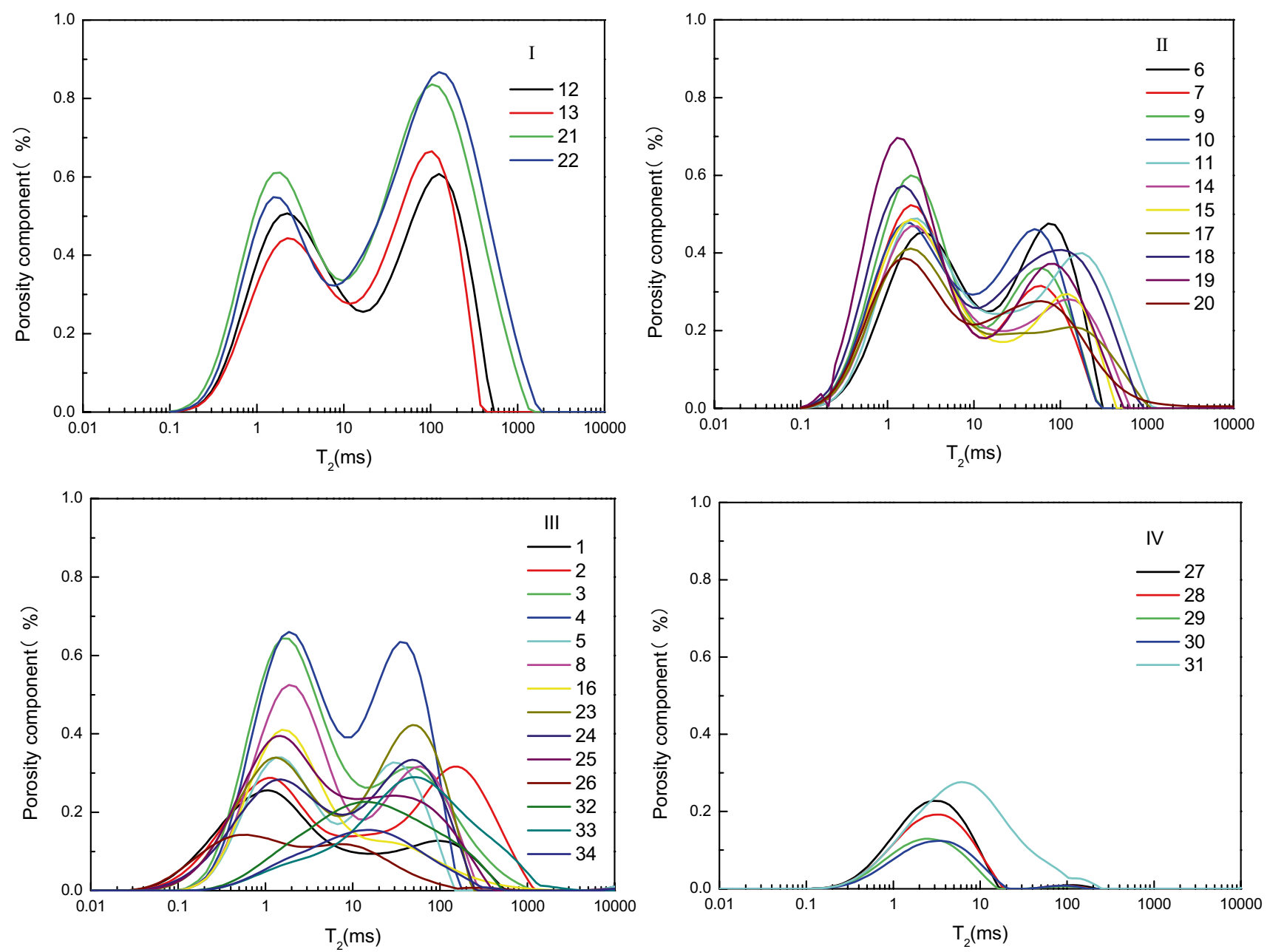

Fig. $16 \mathrm{NMR} T_{2}$ spectrum of rocks with different pore structures
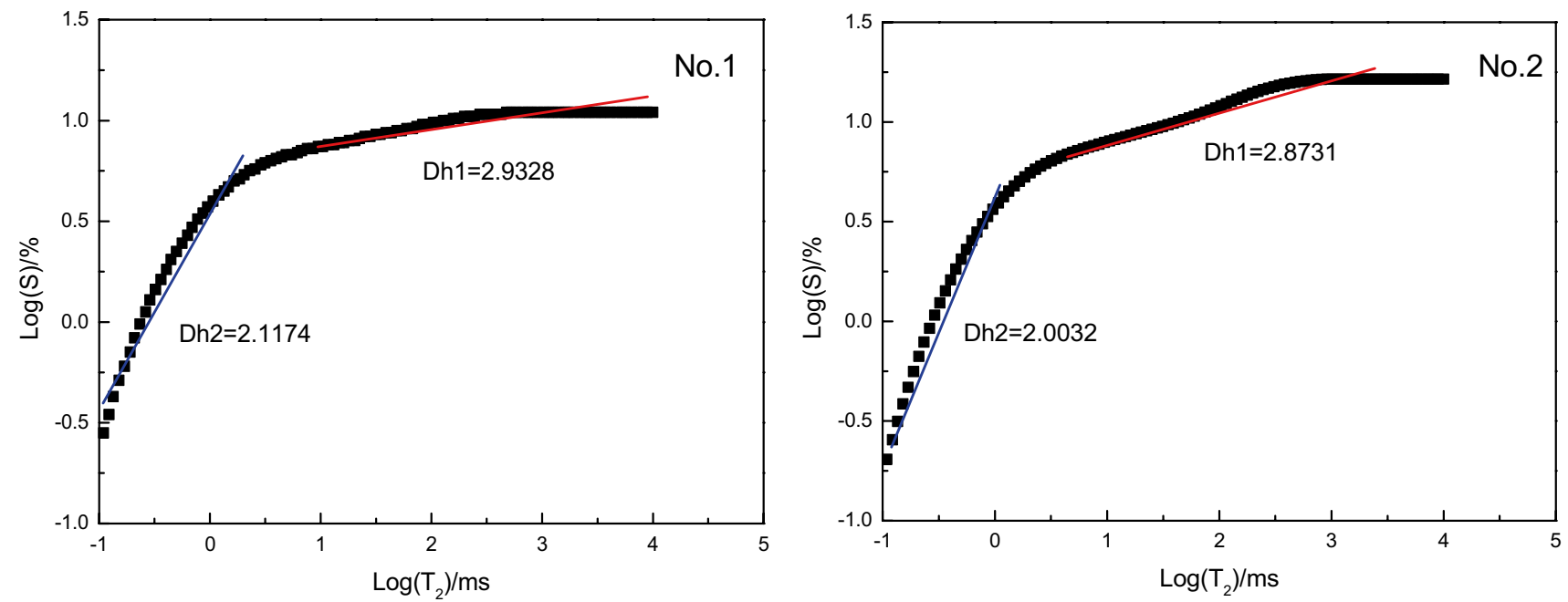

Fig. 17 Nuclear magnetic resonance $T_{2}$ spectrum fractal characteristic curve 

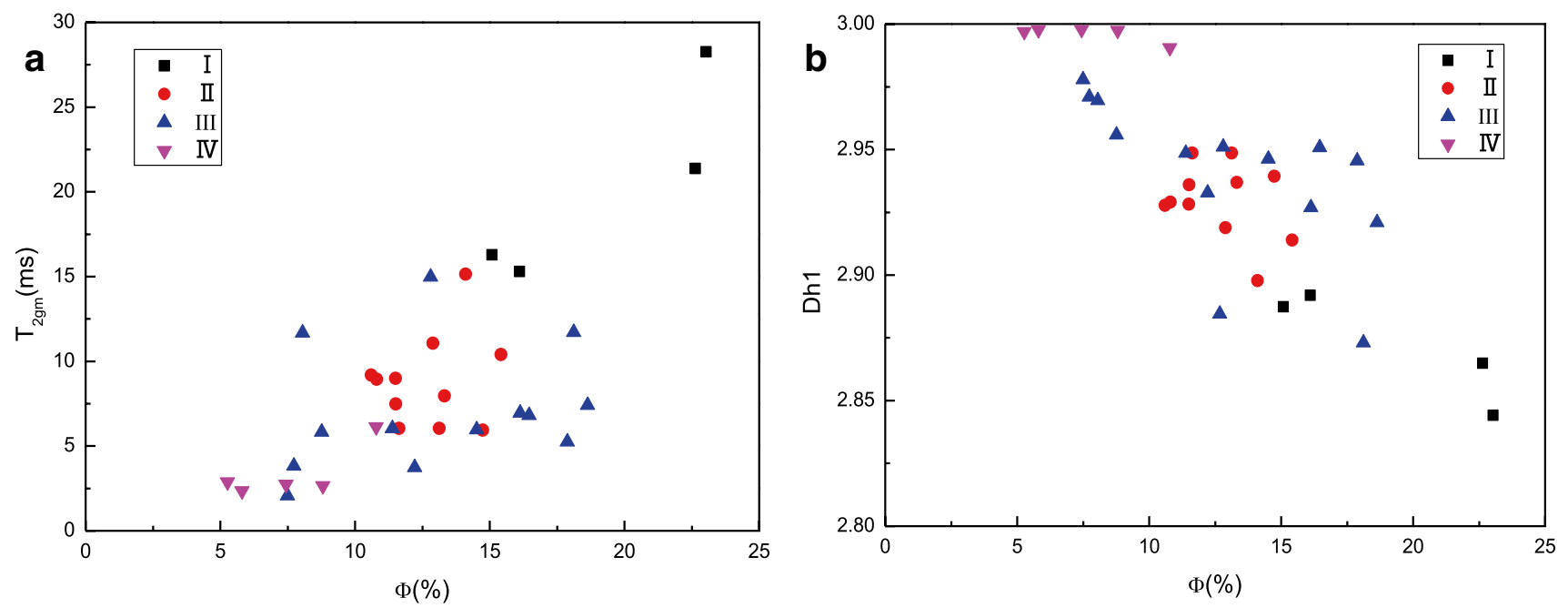

Fig. 18 a Relationship between $T_{2 \mathrm{gm}}$ and $\Phi$ of different pore structures; $\mathbf{b}$ relationship between $D h 1$ and $\Phi$ of different pore structure rocks

Acknowledgements Funding was provided by Natural Science Foundation of China (Grant no. 41174099).

Open Access This article is distributed under the terms of the Creative Commons Attribution 4.0 International License (http://creativeco mmons.org/licenses/by/4.0/), which permits unrestricted use, distribution, and reproduction in any medium, provided you give appropriate credit to the original author(s) and the source, provide a link to the Creative Commons license, and indicate if changes were made.

\section{References}

Biot MA (1956) Theory of propagation of elastic waves in a fluidsaturated porous solid. II. Higher frequency range. J Acoust Soc Am 28(2):179-191

Gao Y, Xiao LZ (2015) An improved TSVD algorithm to inverse NMR relaxation time. Oil Geophys Prospect 50(2):376-381

Ge XM, Fan YR, Yang DG, Hu HC, Deng SG (2011) Study on the influential factors of saturation exponent based on the equivalent rock element theory. Oil Geophys Prospect 46(3):477-481 +488

Guo YH, Pan BZ, Zhang LH, Fang CH (2017) A new model to predict the relative permeability of wetting phase for tight sandstones. Environ Fluid Mech 2:1-13

Guo YH, Pan BZ, Zhang LH, Fang CH (2018) Research and application of the relationship between transverse relaxation time and resistivity index in tight sandstone reservoir. J Petrol Sci Eng 160:597-604

He YD, Mao ZQ, Xiao LZ, Zhang YZ (2005) A new method to obtain capillary pressure curve using NMR T_2 distribution. J Jiling Univ (Earth Sci Ed) 2:007

Kumar M, Han DH (2005) Pore shape effect on elastic properties of carbonate rocks. Seg Tech Program Expand Abstr 24(1):1477

Li H-B, Zhang J-J (2014) A differential effective medium model of multiple-porosity rock and its analytical approximations for dry rock. Chin J Geophys 57(6):835-845

Li HT, Deng SG, Niu YF et al (2018) Study on pore structure classification of low porosity and permeability sandstone. CT Theory Appl 27(5):551-560

Liu T-Y, Tang T-Z, Du H-H, Zhang H-N, Wang H-T (2013) Study of rock conductive mechanism based on pore structure. Chin J Geophys 56(5):674-684
Liu YJ, Li SJ, Wang YG et al (2016) Reservoir prediction based on shear wave in Sulige Gas Field. Oil Geophys Prospect 51(1):165-172

Ma XF, Zhang SC, Lang ZX (2004) Calculation of fractal dimension of pore structure by using subsection regression method. Shiyou Daxue Xueban: Ziran Kexue Ban (J Univ Pet China Nat Sci Ed China) 28(6):54-56

Prasad M (2001) Mapping impedance microstructures in rocks with acoustic microscopy. Lead Edge 20(2):172-179

Shi YJ, Li GR, Zhou JY (2008) Study on litho-electric character and saturation model of argillaceous low-permeability sandstone reservoir. Well Logging Technol 32(3):203-206

Verwer K, Eberli GP, Weger RJ (2011) Effect of pore structure on electrical resistivity in carbonates. AAPG Bull 95(2):175-190

Wei L, Xiao ZX (2009) Comparative studies on methods of evaluation of reservoir pore structure by using nmr (nuclear magnetic resonance) well logging data. Oil Geophys Prospect 44(6):773-778

Wu GM, Li X, Gao SS, An WG (2017) Optimal thresholding in carbonate reservoir CT image binarization based on fractal theory. Oil Geophys Prospect 52(5):1025-1031

Xia L, Wenzhi Z, Cancan Z, Tongshan W, Chaoliu L (2012) Dualporosity saturation model of low-porosity and low-permeability clastic reservoirs. Pet Explor Dev 39(1):88-98

Xiao LZ (1998) Nuclear magnetic resonance imaging logging and rock nuclear magnetic resonance and its application. Science Press, Beijing

Xiao LZ (2012) Nuclear magnetic resonance logging theory and method for complex reservoirs in China. Science Press, Beijing

Zhang LH, Pan BZ, Shan GY, Liu SH, Guo YH, Fang CH (2017) Probability distribution method based on the triple porosity model to identify the fluid properties of the volcanic reservoir in the Wangfu fault depression by well log. Comput Geosci 21(2):241-246

Zhao J, Jiang YZ, Wang WN et al (2003) Investigation of rock pore structure using NMR technology. Well Logging Technol 27(3):185-188

Publisher's Note Springer Nature remains neutral with regard to jurisdictional claims in published maps and institutional affiliations. 